\title{
Theories for Tunneling Microscopy
}

\author{
A.A. Lucas \\ Facultés Universitaires Notre-Dame de la Paix, Namur, Belgium
}

In addition to becoming recognized as one of the most ubiquitous physical phenomena in nature, tunneling has been exploited in a great variety of devices such as beam splitters, microwave attenuators, optical spectrometers, molecular masers, tunnel diodes, Josephson SQUIDS, etc. But probably the most important technical triumph based on tunneling is the recent invention of two new microscopes capable of atomic resolution.

As a theoretical concept, particle tunneling burst onto the scientific scene in 1928 , with the successful application of quantum mechanics to three unexplained phenomena: alpha decay and fusion of nuclei (Gamow), auto-ionisation of hydrogen in a static electric field (Oppenheimer), and field electron emission from metals (Fowler and Nordheim). These problems were treated on the basis of the quantum mechanical penetration or "tunneling" of a particle into a forbidden potential energy barrier. The penetration was a manifestation of the wave nature of the particle postulated only four years earlier by de Broglie.

Today we retrospectively think of tunneling as the more general process by which any wave field penetrates into a forbidden barrier, i.e. a region in which the wave amplitude attenuates exponentially. The wave may represent a mechanical, an electromagnetic or a mixed classical vibration, or it can be a quantum wave associated with a single degree of freedom or "particle" (elementary or compound). Thus, tunneling is now understood to occur in the realm of classical acoustics and optics as well as in all subfields of quantum physics.

One speaks of tunneling whether or not the wave traverses completely the forbidden barrier. In field electron emission from metals, the forbidden region is very thin and separates the incident and transmission regions. In the optical phe-

Professor Amand Lucas is with the Department of Physics, Facultés Universitaires N.-D. de la Paix, Rue de Bruxelles 61, B-5000 Namur. He graduated and received his Doctorate, in 1956, from the University of Liege and then worked in the USA, at Battelle and IBM, and as a visiting scientist at the ICTP, Trieste and ESTEC, Nordwijk. He also chairs the Interuniversity Research Project in Interface Science. nomenon of total internal reflection, the wave only partly penetrates a thick forbidden zone and is reflected back into the same, incident region.

\section{Tunneling Microscopy}

Field-ion microscopy (FIM), developed in the early fifties, gave us the first direct, "visual" proof of the atomic constitution of matter. Then, in the early eighties, scanning tunneling microscopy (STM) was introduced [1]. Whereas FIM was conceived and developed using simple physical principles, the unexpected discovery of STM was the spectacular result of technical advances in controlling the relative positions of macroscopic bodies with nanometric precision.

Triggered by the success of STM, several other scanning microscopies quickly appeared including an optical version called scanning near-field optical microscopy (SNOM) [2]. This technique, also based on wave tunneling, produces images at intermediate, mesoscopic resolution but under conditions far less stringent than the high vacuum environment of transmission or scanning electron microscopy.

In both STM and SNOM, an "atomic tip" held on a substrate (refered to hereafter as the microscope head) is made to scan the proximity of an object (Fig. 1). In STM, the tip is a real atom or a small atomic cluster at the apex of a needle. In SNOM, the tip may consist of a speck of matter of submicron size resting on a flat substrate or it may be a small aperture hole in an opaque screen [2]. The waves (electrons or photons, respectively) tunnel from the substrate, via the "atom" and empty space, into the object (Fig. 1). The transmitted wave intensity which varies according to the topographic and other corrugations of the object surface, is monitored during the scan and is converted to form an image of the surface.

Instruments are often operated in the constant-intensity mode where what is recorded is the electronic signal applied to the piezoelectrically controlled microscope head in order to maintain the total tunnel current or intensity at a preset value. Without the tip, there is no useful image in that the weaker tunneling intensity in the absence of the tip or far away from it is considered as noise.

A large amount of experimental information has already been accumulated in the last few years, especially with STM. By contrast, the harvest of theoretical results is rather meager: this is a field where experiment is far ahead of theory.

In the present paper we shall be mainly concerned with aspects of the theory of imaging in STM as being representative of microscopies based on quantum tunneling. A short review of related theory for SNOM imaging, representative of microscopies based on classical tunneling, is given in the Appendix.

\section{Tunneling Theory}

Broadly speaking, the mathematical basis for discussing classical tunneling or single particle (nonrelativistic and nonsuperconductive) quantum tunneling in the microscopes is a generalized wave equation

$$
\left[\Delta+\boldsymbol{k}^{2}(\boldsymbol{r})\right] \Psi(\boldsymbol{r})=0
$$

where $\Delta$ is the Laplacian operator, $\Psi$ is

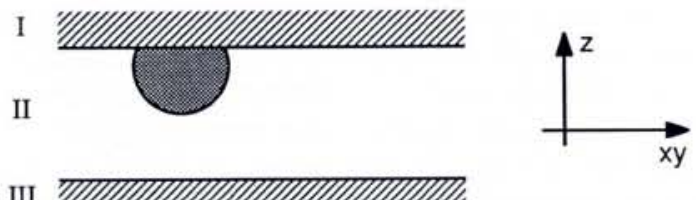

Fig. 1 - Schematic representation of a tunnel junction in STM and SNOM microscopies. Waves travel in the z-direction from a substrate through an atom or a spherical cluster of atoms supported by the substrate and into a small volume of empty space before entering the surface, in the xy-plane, of the object under examination. The junction is divided into three regions: I-in the substrate, II-in the empty space, and III-in the object. 
a scalar or vector wavefunction, and $\boldsymbol{k}$ is the local wavevector related to the total energy of the wave and to the potential energy barrier. It corresponds to the Schrödinger equation for STM and the vector Helmholtz equation for SNOM.

Space is divided into three regions (Fig. 1). In the allowed region $\mathrm{I}, k^{2}>0$ and the wave $\Psi \approx \Psi_{0}(\boldsymbol{r}) \exp (i \boldsymbol{k} . \boldsymbol{r})$ propagates freely from the source towards both the microscope head and region II. In forbidden region II, $k^{2}<0$ (or, more generally, $k$ is complex) so that the solution of (1) has an exponential attenuation factor. In region III which begins with the surface under examination, $k^{2}$ is again positive and the attenuated wave propagates freely and can be detected. (In SNOM, waves backscattered into region I may also be used [2].)

The procedure consists in calculating, for a given position of the microscope head relative to the surface, the tunneling barrier in $k^{2}(r)$ and all the relevant wavefunctions $\Psi(r)$, namely those emitted by the source and collected by the detector. From these, the quantum mechanical current density

$$
j(\boldsymbol{r}) \approx \Sigma \mathrm{Im}_{\Psi}\left[\Psi(\boldsymbol{r}) \operatorname{grad} \Psi^{*}(\boldsymbol{r})\right]
$$

and the total electron current in STM, or the classical Poynting vector and the scattered light intensity in SNOM, can be determined.

In this short review we intend to restrict the discussion to the present understanding of the remarkable resolving power of the microscopes. A broader review of theoretical aspects for STM can be found in [3]. Concerning SNOM, several references to relevant theoretical works are cited in [2] but the general theory is still relatively sketchy (see the Appendix).

\section{Resolution}

The resolution of a microscope is the smallest distance between two physical features, suitably defined, on the surface of the object at which separate images will form. One speaks of the "vertical" resolution (in the $z$-direction in Fig. 1) as distinct from the lateral resolution ( $x y$-directions). The two are related since both stem from modulations of the shape of the tunnel barrier. They depend only indirectly on the wavelength $\lambda$ of the radiation used.

Unlike ordinary light microscopies, the resolution need not be limited to features separated by distances larger than $\lambda$. Indeed, the vertical resolution of both STM and SNOM is a tiny fraction of $\lambda$ because tunneling is extremely sensitive to the tip-surface distance. It is estimated that altering this distance in STM by $0.1 \mathrm{~nm}$ changes the total tunnel current by nearly an order of magnitude, thus allowing vertical control of the tip position to better than $0.01 \mathrm{~nm}$. This in turn implies that the lateral resolution may also be much smaller than $\lambda$ [4], becoming less than $0.2 \mathrm{~nm}$ with electrons of $0.5 \mathrm{~nm}$ wavelength (i.e. the Fermi wavelength of metals used as a tip). In SNOM, features at least twenty times smaller than visible wavelengths can be resolved [2], far beyond the diffraction limit of an optical microscope.

The reason for the superb resolution in these so-called near-field microscopies is the positioning of the object under examination within a distance from the tip on the order of the wavelength of the incident radiation (in ordinary light microscopies, the detector is positioned in the far-field zone and additional, aberration-prone lenses are needed to focus the radiation and magnify the image of the scatterer). Scanning the tip over a surface corrugation modulates the intensity of the waves tunneling into the near-field region and hence of the intensities of transmitted waves and the detected signal. Under these conditions, the resolving power of the instrument is limited by the size of the tip, while the distance of the tip from the surface controls the signal-to-noise ratio. The minimum tip size in STM is one atomic radius whereas in SNOM the size of the particle used as the tip may go down to a few tens of nanometres, notwithstanding the necessity to maintain useful contrast.

\section{Transfer Hamiltonian Theory}

The analysis of early STM data relied on ideas of one-dimensional tunneling taken from textbooks where, for a square barrier, the tunnel current depends on the barrier width $d$ and height $\phi$ (the work function, typically $\approx 4 \mathrm{eV}$ for metals) according to an approximate exponential law,

$$
I \approx \exp (-2 \kappa d)
$$

where $\kappa=\hbar^{1}(2 m \phi)^{1 / 2} \approx 10 \mathrm{~nm}^{-1}$ is the inverse of the decay length of the wavefunctions in the forbidden barrier. However, by virtue of being near-field microscopies, STM and SNOM require a fully 3-d tunneling theory. Moreover, the peculiar shape of the tip and the smallscale corrugation of the object surface remove all continuous or discrete spatial symmetries which would make the wave equation (1) separable. Without such symmetries there is no exact $3-d$ theory which can be easily implemented on a computer.

The first attempts to go beyond the elementary ideas of the 1-d theory relied on the so-called transfer Hamiltonian (TH) formulation of tunneling due to
Bardeen. This is a fully $3-d$ approach but, being derived from first order perturbation theory, it strictly applied only to the thick barrier limit where the interaction between tip and surface is "sufficiently" weak. Indeed, the zeroorder wavefunctions required to compute the tunnel current in the first order are taken as those for infinitely distant, noninteracting electrodes. In an ordinary metal-oxide-metal tunneling junction, the planar oxide barrier is at least $2 \mathrm{~nm}$ thick. In STM, to obtain atomic resolution with sufficient contrast it is necessary to approach the object surface with the tip to a distance of less than an estimated $1.0-1.5 \mathrm{~nm}$, i.e. 3 or 4 times the interatomic spacings in solids.

From the decay length $\kappa^{-1} \approx 0.1 \mathrm{~nm}$ of the wavefunctions indicated by $1-d$ arguments, it is expected that these are "safe" distances for the TH approximation to hold. However, there is no way to ascertain quantitatively the distance at the lower limit of validity of the TH formulation without a comparison with the solution for an exactly soluble model problem. The new theoretical approach based on scattering which we shall discuss later provides such a solution.

The goal in STM is to obtain information on the atomic and electronic structures of the isolated object surface. Operating the microscope with a thick barrier is highly desirable because the wavefunctions of the surface are unperturbed by the tip and they can be simulated using the sophisticated methods of theoretical surface physics. But the thick barrier approximation does not remove the problem of simulating the wavefunctions of the tip with its awkward geometry. This problem has been tackled in several ways: by treating the tip as a source of one single s-wave [5], by modelling it as a single atom adsorbed on a flat jellium substrate [6], etc.

\section{S-wave model}

The $s$-wave tip approach has the advantage of reducing the $\mathrm{TH}$ expression for the total tunnel current to a simple analytical form:

$J \approx \Sigma_{\mathrm{v}}\left|\Psi_{\mathrm{v}}\left(r_{\mathrm{t}}\right)\right|^{2} \delta\left(E_{\mathrm{v}}-E_{\mathrm{F}}\right)=\rho\left(r_{\mathrm{t}}, E_{F}\right)$ written here for zero temperature and small bias voltage $V$ between the electrodes. Result (2) has the familiar form of a spectral density, depending on the wavefunctions $\Psi_{v}$ of the surface for energy $E_{v}$ at the Fermi level $E_{\mathrm{F}}$ (the only states involved in current transport by tunneling for $V \approx 0$ ) and evaluated at the centre $r_{t}$ of the spherical tip (the point source of $s$-waves) [5]. $\rho\left(r_{t}, E_{F}\right)$ is just the local density of states (LDOS) which directly reflects the atomic and electronic structures of the surface. 
Formula (2) has been up to now the workhorse for interpreting much of the STM data for both clean and adsorbatecovered surfaces. This simple result also lends itself to a quantitative evaluation of the spatial lateral resolution of STM [4]. It is found that, for the case of metal surfaces, the resolution function (which determines the image via a linear convolution of the object structure) is approximately independent of the surface and is a simple gaussian of rms width $\left(z_{0} / 2 \kappa\right)^{1 / 2}$ where $z_{0}$ is an average distance from the tip to the surface. For example, $z_{\mathrm{O}} \approx 1.2 \mathrm{~nm}$ and $\kappa$ $\approx 10 \mathrm{~nm}^{-1}$ gives a resolution width of $0.25 \mathrm{~nm}$.

A resolving power of this amount is adequate for imaging individual atoms if the surface LDOS has sufficient spatial contrast at the tip centre. This is generally not the case for bare metal surfaces on account of the smoothness of their delocalized conduction electron densities. Indeed, only the more pronounced corrugations associated with extended defects such as atomic steps or surface reconstructions are usually observed on clean metal surfaces when operating with the tip at $1 \mathrm{~nm}$ or more from the surface. The resolving power is sufficient, however, to image individual atoms and groups of atoms pertaining to molecules adsorbed on metal surfaces [7]. The observed molecular images correlate quite well theoretical simulations based on (2) and computed using the valence molecular orbitals of the adsorbate.

For semiconductors, the LDOS has a deeper corrugation associated with localized covalent bonds. Atom visibility is also further enhanced by reconstruction such as occurs on the famous Si(111) $7 \times 7$ surface. It turns out, however, that the resolution function derived from (2), contrary to the metal case, depends rather critically on details of the electronic structure near the edges of the semiconductor band gap. This makes the STM lateral resolution both sample and face dependent [4] so the observed images may not give any direct indication of the atomic positions in the surface unit cell. Thus, on the basis of Eq. (2), it has been possible to understand the origin of the anomalously large apparent corrugations on some semiconductors such as $\mathrm{GaAs}$ and on semimetals such as graphite [4].

A major criticism of the $s$-wave tip model is that it largely ignores the atomic and electronic structures of the tip. By comparing the results of the $\mathrm{TH}$ theory applied to two simple models of a polyatomic, spherical tip (whose atoms were treated either as coherent or as incoherent $s$-wave electron sources), Tersoff argues that the simple LDOS expression given in (2) continues to hold under more general conditions than was originally assumed.

\section{Atom-on-jellium model}

The other main approach for evaluating the effect of tip wavefunctions is the atom-on-a-jellium model [6]. The TH expression for the tunnel current is also used but the tip electronic structure receives a more exact treatment than in the $s$-wave model: it is calculated, by density-functional methods, as that of a real atom in the presence of an uncorrugated jellium substrate. The sample surface is also treated using the flat jellium approximation, although it may also support a single adatom. The method is then better suited for discussing certain important aspects of the current flow in STM such as the role of valence resonances of the tip adatom, the bias dependence of the current intensity through an adsorbed atom as observed in STS (scanning tunneling spectroscopy), the apparent "vertical" size of the surface adatom, etc.

The lateral resolution can be evaluated in this approach from the spatial distribution of the tunnel current in an intermediate region between the tip atom

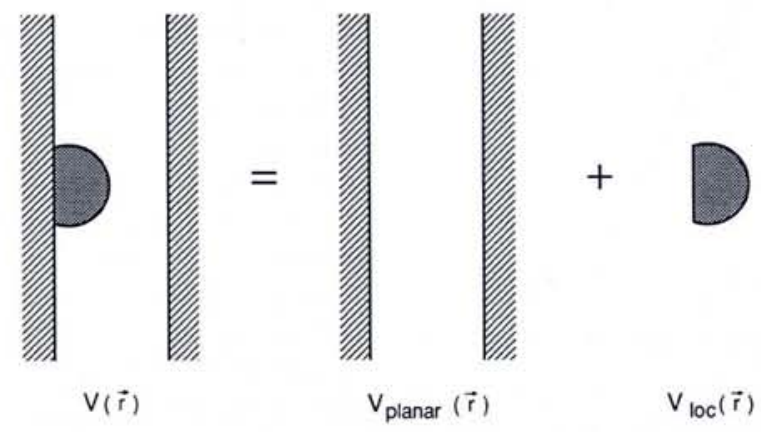

Fig. 2 - Symbolic decomposition of the tunnel barrier to allow the application of exact methods of scattering theory to treat three-dimensional barriers. The barrier voltage is split into a planar part and a localised component, due to the tip atom or cluster of atoms, for perturbational treatment. and the object surface, where the wavefunctions are required to calculate the tunneling matrix elements of the $\mathrm{TH}$ theory. Lateral resolutions of the order of observed ones and consistent with those predicted by the $s$-wave tip model are obtained.

\section{Scattering Formulation}

At large tip-surface separations the resistance of an STM junction at a metal surface is typically $10^{9} \Omega$. Under a bias of $1 \mathrm{~V}$ between tip and surface, this represents a tunneling current of $1 \mathrm{nA}$ and hence an average residence time of $1 \times 610^{-10} \mathrm{~s}$ for the tunneling electrons on the tip wavefunction. The corresponding level broadening given by the Heisenberg uncertainty principle is very small and the tip wavefunctions should only be weakly perturbed by tunneling, making the $\mathrm{TH}$ approximation legitimate.

One does not know the absolute tipsurface separation in such conditions but is is estimated to be around $1.2 \mathrm{~nm}$. At the other extreme, when the tip atom is in close contact with the surface, at say $0.2 \mathrm{~nm}$, the tip energy levels are massively broadened and shifted by a hopping matrix element of several $\mathrm{eV}$. The experimental contact resistance drops at this point to $10^{4} \Omega$, corresponding to a current of $100 \mu \mathrm{A}$ under a $1 \mathrm{~V}$ bias.

Thus, over a distance variation of 1 $\mathrm{nm}$, the tunnel current changes by five orders of magnitude, as does the perturbation of the tip's energy levels as a result of the increasing overlap of the tip and surface wavefunctions. The $\mathrm{TH}$ thick barrier approximation must therefore break down as a quantitative theory somewhere in the intermediate region, perhaps around 0.5 or $0.6 \mathrm{~nm}$ separation which is the most desirable distance for operating STM with optimum resolution and contrast.

In an effort to remove the weak coupling approximation in STM and other tunneling problems, we have constructed a new theory of one-electron elastic tunneling designed to treat 3-d, nonseparable barriers [8]. Our approach uses the exact methods of scattering theory for localized potentials. We have also confirmed the accuracy of the new method by solving directly the Schrödinger equation via the method of finite elements [9].

The basic ideas are as follows: as illustrated schematically in Fig. 2, the total tunneling barrier is split into a planar component (without the tip apex) and a perturbed component due to the tip atom or cluster of atoms:

$$
V(r)=V_{\text {planar }}(r)+V_{\text {loc }}(r)
$$




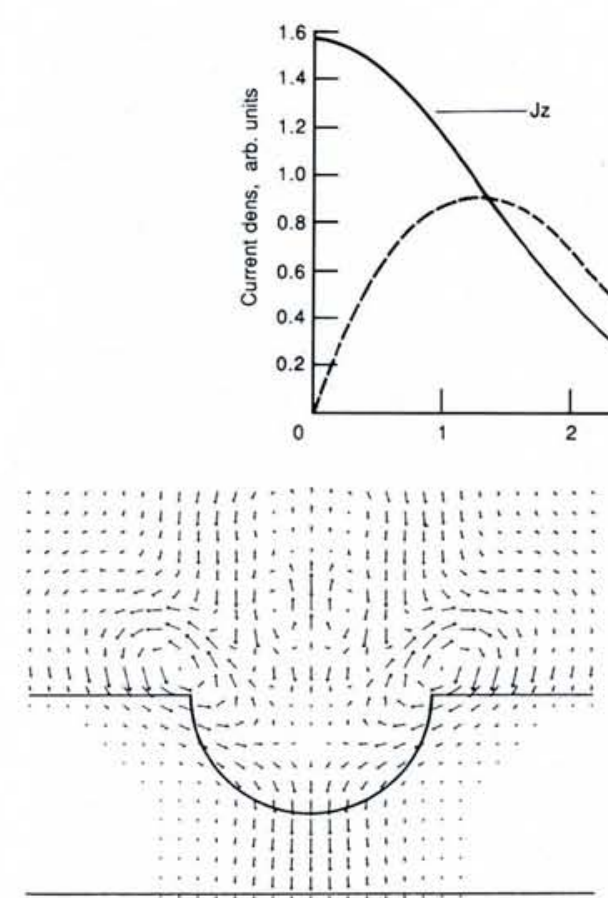

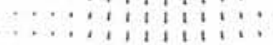

$\ldots, 1,1, \ldots, \ldots$

Discrete or continuous translational invariance parallel to the surface is assumed for the planar part which can, in principle, then be solved exactly by well developed methods borrowed from the theory of planar interfaces. The additional barrier associated with the tip is assumed to be localized in a small spatial region through which most of the tunneling occurs. This feature allows us to treat the tip barrier to all orders of perturbation.

If we call $\Phi(\boldsymbol{r})$ the planar tunneling wavefunctions, the exact wavefunctions $\Psi(r)$ of the total barrier are given by the Lippmann-Schwinger equation

$\Psi(\boldsymbol{r})=\Phi(\boldsymbol{r})+\int \mathrm{d} \boldsymbol{r}^{\prime} G\left(\boldsymbol{r}, \boldsymbol{r}^{\prime}\right) V_{\text {loc }}\left(\boldsymbol{r}^{\prime}\right) \Phi\left(\boldsymbol{r}^{\prime}\right)$ which involves the exact Green function $G\left(r, r^{\prime}\right)$ of the total barrier. The latter is given by a Dyson equation

$G\left(r, r^{\prime}\right)=\int \mathrm{d} r^{\prime \prime}\left[\left(1-\mathrm{g} V_{\text {loc }}\right)^{-1}\right]_{r, r^{\prime \prime}} \mathrm{g}\left(r^{\prime \prime}, r^{\prime}\right)$ in terms of $V_{\text {loc }}$ and the exact Green function $g\left(r, r^{\prime}\right)$ of $V_{\text {planar. The }}$. The integral equations can be transformed into discrete matrix equations for $r$ and $r^{\prime}$ on a $3-d$ grid of points covering the localized tunneling region [8]. The computational feasibility of the discretization scheme hinges on the fact that $V_{\text {loc }}$ is localized and that the tunneling wavefunctions are required only in the region of finite dimensions covering the tip, i.e. at a computationally manageable number of grid points.

Implementation of the general scheme is naturally quite formidable. Owing to the atomic structure of the tip substrate, the planar problem is already more complex than a full LEED (low can include the many-body correlation effects represented classically by the self-interaction of the tunneling electron with its multiple images in the metal electrodes. The model has continuous axial symmetry which, in effect, reduces the problem to a discrete set of independent 2-d tunneling problems, one for each axial angular momentum of the wavefunctions.

Typical results for the spatial distribution of the tunneling current are shown in Fig. 3. These are the first current density maps for a nontrivial multidimensional tunneling problem solved without approximation. Stationary eddy currents appear to develop inside the tip electrode (Fig. $3 \mathrm{~b}$ and similar pictures in [9]) due to diffraction of the electron plane waves by the hemispherical tip. Their spatial distribution turns out to be very sensitive to the geometrical parameters of the junction but one does not know if they will persist in the presence of an underlying atomic corrugation.

In Fig. 3a, the tunneling channel has a lateral spread (which can be taken as its full width at half maximum) on the surface that is significantly smaller than the radius of the tip protrusion. From this one expects that lateral corrugation of the surface on a scale smaller than the tip radius would be resolvable. These results confirm, from first principles, the atomic resolving power of STM. It was also found that the beam width increased only slowly with the tip radius $(0.2 \mathrm{~nm}$ per $\mathrm{nm})$ and was weakly dependent on the tip-surface distance. This last result is consistent with the weak $\sqrt{ } z_{0}$ behaviour of the theoretical resolution width for the $\mathrm{TH}$ approach.

\section{Muffin-tin potentials}

A realistic representation of the surface using localized Green functions has recently been implemented, with the atomic corrugation of the surface electrode fully taken into account via muffin-tin potentials, but keeping the same free-electron, hemispherical tip. The Green function for the planar junction (without the tip) was generated using suitably adapted, state-of-the-art LEED programs. The approach was applied to the $(100)$ surface of palladium. One of the interesting results, shown in Fig. 4, was that the difference between tunneling from above an atom and from a bridge position of the tip between two surface atoms was rather small for this metal, confirming the findings of the $\mathrm{TH}$ theory for metal surfaces [4].

The last step in improving the model, namely the inclusion of a realistic atomic structure for the tip electrode, is being developed at present. 


\section{Conclusions}

By avoiding the uncertainties attached to the thick barrier approximation of the TH tunneling theory, the scattering method promises to give us a tool to better understand the tunnel barrier itself and the underlying electronic structure probed by STM. However, with presently available computer power, it should be said that the implementation of the localized Green function method is very time consuming so we are fortunate in having a simple interim formula such as (2) for the interpretation of images obtained at least in the thick barrier approximation.

The development of STM theory is likely to follow a course similar to that of LEED over the last 20 years where improving methodology and enhanced computer power slowly brought the theory to its present predictive capacity.

\section{REFERENCES}

[1] Binnig G. and Rohrer H., Helv. Phys. Acta 55 (1982) 726 .

[2] Pohl D.W., Fisher U.Ch. and Dürig U., SPIE 897 (1988) 84; Fisher U.Ch. and Pohl D.W., Phys. Rev. Lett. 62 (1989) 458.

[3] Feuchtwang T.E. and Cutler P.H., Physica Scripta 35 (1987) 132.

[4] Tersoff J., Phys. Rev. 39 (1989) 1052. [5] Tersoff J. and Hamann D., Phys. Rev. B31 (1985) 805.

[6] Lang N., Phys. Rev. Lett. 55 (1985) 230; Phys. Rev. B34 (1986) 5947; Phys. Rev. Lett. 58 (1987) 45.

[7] Lippel P.H. et al., Phys. Rev. Lett. 62 (1989) 171.

[8] Lucas A.A. et al., Phys. Rev. 37 (1987) 1070.

[9] Laloyaux Th. et al., J. Microscopy 152 (1988) 53.

[10] Dereux A., Vigneron J.-P., Lambin Ph. and Lucas A.A., Phys. Rev. B38 (1988) 5438.

\section{Appendix:}

Theory for Light Wave Tunneling in SNOM

SNOM exploits the existence of an important electromagnetic power density which tunnels into the near-field region of the "tip" extending towards the surface [2]. As mentioned above, the tip may be a small particle less than $100 \mathrm{~nm}$ in size. When the material of the particle is suitably chosen, e.g. gold, its polariton eigenmodes can further enhance the field of the scattered light waves at a particular resonance frequency in the visible, rather like in a spectroscopic technique called surface enhanced Raman scattering. The evanescent radiation is coupled out of the tip region by the presence of the surface and can be collected and photomultiplied, either in transmission or in reflection, via an auxiliary light microscope focused onto the tip area. Owing to the tunneling effect, reinforced by an eventual resonance condition, the intensity is very sensitive to the proximity and dielectric properties of the surface under examina- tion. The scattered light can be monitored during the scan in a constant-distance or constant-intensity modes.

A theory for SNOM demands the solution of Maxwell's equations in an awkward geo metry devoid of any spatial symmetry. Very special cases such as scattering by an iso lated sphere, by a very small hole in a thin opaque film, by a sphere on a flat substrate, etc. have been solved either exactly or approximately but the results are only marginally useful for the SNOM situation with its full complexity.

The analogy between the theoretical problems posed by SNOM and STM is as striking as the similarity between the experimental configurations of the two microscopes. Their common characteristic is that propagation and tunneling of waves through a stratified, planar medium are modified by a strong but spatially localized perturbation associated with the tip. Apart from changes of scale by several orders of magnitude of the size of the tip $(<100 \mathrm{~nm}$ $v s<1 \mathrm{~nm}$ ) and the wavelength $(500 \mathrm{~nm}$ vs $0.5 \mathrm{~nm}$ ), the major difference lies in the vec tor nature of the Helmholtz wave equation versus the scalar Schrödinger equation. This lead us to formulate a new, computationally feasible approach for the prediction of SNOM intensities based on the technique of localized Green functions similar to that exploited in our STM theory [8].

The starting point is the calculation of the unperturbed electromagnetic tensor Green function for the polaritons of a planar stack of dielectrics - metallic, vacuum and surface layers - typical of a SNOM experiment [2]. This problem has already been solved exactly in the context of infrared and attenuated total reflection spectroscopies of arbitrary multilayer materials [10]. The planar Green function can then be used to construct the exact Green function and the scattered wavefields of the system in the presence of the tip whose perturbation is treated to all orders by a spatial discretization of the electromagnetic LippmannSchwinger and Dyson equations.

Just as in our scattering solution of the STM problem, the effects of the tip and of the surface corrugation are represented by a localized dielectric "barrier" $\varepsilon_{\mathrm{loc}}(r, \omega)$ embedded in a planar medium of trans lationally invariant dielectric function $\varepsilon_{\text {planar }}$ $(z, \omega)$. The advantage of this approach is its ability to handle a tip of any shape and composition (homogeneous or not) provided its spatial extent is roughly equal to or smaller than the wavelength, which is just the requirement of good performance for a nearfield microscopy such as SNOM.

The method is applicable to magnetic "barriers" where the inhomogeneities are in the corrugation of the magnetic susceptibility $\mu_{\text {loc }}(r, \omega)$. Owing to the vector nature of the electromagnetic field, the matrices expressing the discretized integral equations of SNOM are just three times as big as in STM, but one gains on several counts. For instance, there is only one single incident plane wave in SNOM (instead of the many Fermi level states in STM) and since the tip size, measured on the scale of the wavelength, is substantially smaller than in STM, the discretization grid can be coarser.

The numerical codes to implement this scheme are now being developed and applied to test problems as well as to real cases encountered in practice [2].

\section{Acknowledgements}

The author is grateful to Th. Laloyaux, A. Dereux, I. Derijcke, J.-P. Vigneron, Ph. Lambin, M. Taborelli for discussions and comments on the manuscript. Part of the work was developed under the Belgian Interuniversity Research Programmes of the Prime Minister's Office, Science Policy Programming.

\section{Experimental Nuclear Physics in Portugal}

The Centre for Nuclear Physics invites applications for a five-year tenure-track position at the rank of Associate Scientist

to develop in Portugal an experimental group in nuclear physics.

Tenure will be considered after three years on the job. A Ph.D. is required. Experience in either heavy ion physics, nuclear spectroscopy, electron or hadronic physics is expected. A successful candidate is also expected to collaborate and carry out experiments at major European or North American facilities and be responsible for the submission of projects to the Portuguese funding agencies and the EEC Science Program to obtain the necessary funds to carry out his research.

Portugal is in a particularly good position to receive major support from the EEC to develop high quality research groups. The candidate is therefore expected to have the qualities of a leader with a strong commitment to the training of young graduate students and postdocs.

The Centre for Nuclear Physics has a strong theoretical group in nuclear reactions and few-body physics and a very successful experimental program in nuclear solidstate physics, atomic inner-shell studies and archeometry.

The salary depends on experience and lies in the range \$US 20000.- 25000.-/year, free of taxes.

Portugal is one of the least expensive countries in Europe, and Lisbon is an attractive city with a mild climate and very pleasant surroundings.

Send applications, including a curriculum vitae and at least five references and nominations to: Prof. A.C. Fonseca, Centro de Física Nuclear, Av. Gama Pinto 2, 1699 Lisbon, Portugal. 